\title{
CADM1 promotes adhesion to vascular endothelial cells and transendothelial migration in cultured GIST cells
}

\author{
JIAYIN YUAN $^{1}$, TAKAKO KIHARA ${ }^{1}$, NEINEI KIMURA ${ }^{1}$, TAKASHI YAMASAKI ${ }^{1}$, \\ MAKOTO YOSHIDA $^{1}$, KOJI ISOZAKI ${ }^{1}$, AKIHIKO ITO ${ }^{2}$ and SEICHI HIROTA ${ }^{1}$ \\ ${ }^{1}$ Department of Surgical Pathology, Hyogo College of Medicine, Nishinomiya, Hyogo 663-8501; \\ ${ }^{2}$ Department of Pathology, Faculty of Medicine, Kindai University, Osaka-Sayama, Osaka 589-8511, Japan
}

Received September 17, 2021; Accepted December 20, 2021

DOI: 10.3892/ol.2022.13206

\begin{abstract}
Gastrointestinal stromal tumor (GIST) is the most common mesenchymal tumor of the human gastrointestinal tract. Small intestinal GISTs appear to be associated with poorer prognosis and higher metastasis rate than gastric GISTs of the same size and mitotic index. Recently, we reported that cell adhesion molecule $1(C A D M I)$ is expressed specifically in most small intestinal GISTs, but not in most gastric GISTs, suggesting that this difference in $C A D M 1$ expression between gastric GISTs and small intestinal GISTs might influence the difference in clinical behavior between them. The aim of the present study was to examine whether high $C A D M 1$ expression affected proliferation, migration, invasion, adhesion to endothelial cells and transendothelial migration of cultured GIST cells by comparing original GIST-T1 cells with very low CADMI expression with GIST-T1 cells with high CADM1 expression induced by $C A D M 1$ cDNA transfection (GIST-T1-CAD cells). GIST-T1-CAD cells had reduced ability to proliferate, migrate and invade compared with the original GIST-T1 cells, but showed significantly higher ability to adhere to human umbilical vein endothelial cells and migrate through endothelial cell monolayers. Thus, CADM1 may contribute to higher metastasis rates in small intestinal GISTs facilitating tumor cell adhesion to vascular endothelial cell and transendothelial migration of tumor cells. CADM1 might serve as a potential target for inhibition of metastasis in small intestinal GISTs.
\end{abstract}

\section{Introduction}

Gastrointestinal stromal tumor (GIST) is the most common mesenchymal tumor of the human gastrointestinal (GI)

Correspondence to: Dr Seiichi Hirota, Department of Surgical Pathology, Hyogo College of Medicine, 1-1 Mukogawa-cho, Nishinomiya, Hyogo 663-8501, Japan

E-mail: hiros@hyo-med.ac.jp

Key words: gastrointestinal stromal tumor, cell adhesion molecule 1, proliferation, adhesion to vascular endothelial cells, transendothelial migration tract (1). GISTs occur throughout the GI tract, but most of them arise in the stomach (60-70\%) or small intestine (20-30\%) (2). Activating mutations in the c-kit gene and PDGFRA gene, encoding KIT tyrosine kinase and platelet-derived growth factor receptor tyrosine kinase, respectively, are considered the main oncogenic drivers of GIST (3). The minority of GISTs harboring neither c-kit gene nor PDGFRA gene may have mutations in the $N F 1, B R A F$, or $S D H$ complex genes (4-6).

GISTs are neoplasms with malignant potential varying from virtual indolence to rapid progression. Up to $20 \%$ of GIST patients have overt metastases at diagnosis, and the metastases typically occur in the abdominal cavity or the liver. Small intestinal GISTs are considered to have a worse prognosis than gastric GISTs because of their higher risk of metastasis and tumor-related death (7). Recent research has shown that small intestinal GISTs exhibit more aggressive features such as high pathological grade and large size than gastric GISTs (8). Distinct transcription profiles related to the anatomical location of GISTs have also been reported previously (9). The results of hierarchical clustering analysis of the transcripts showing that GISTs, roughly divided into two groups such as gastric GISTs and small intestinal GISTs, may partially account for the more aggressive behavior of small intestinal GISTs than gastric GISTs of similar size and mitotic rate. Thus, the widely used risk classifications for GIST metastasis such as the Armed Forces Institute of Pathology (AFIP) classification and modified Fletcher classification include tumor location as a risk factor.

We have recently reported that cell adhesion molecule 1 $(C A D M 1)$ is expressed specifically in most small intestinal GISTs but not in most gastric GISTs (10). CADM1 is a member of the immunoglobulin superfamily that was initially known as spermatogenic immunoglobulin superfamily (SgIGSF) and synaptic cell adhesion molecule (SynCAM) (11). CADM1 is also a tumor suppressor of lung cancer (TSLC1) (12). Loss of $C A D M 1$ expression probably due to methylation of the $C A D M 1$ gene promoter is frequently found in various types of epithelial cancer, such as gastric cancer, breast cancer, and esophageal squamous cell carcinoma (13-15). Therefore, CADM1 in those cancers is considered to act as a tumor suppressor. In contrast, CADM1 appears to be a tumor promoter in adult T cell leukemia/lymphoma (ATLL) (16) and acute myelocytic leukemia $(17,18)$, which show high $C A D M 1$ expression with 
enhancement of tumor growth, tissue invasion by the tumor cells, and tumor cell adhesion to the vascular endothelium (18). Recent research also reported that $C A D M 1$ is highly expressed in $\sim 80 \%$ of small cell lung cancers (SCLCs) and promotes malignant features of them $(19,20)$.

In the present study, we examined whether CADM1 affects proliferation, migration, invasion, adhesion to endothelial cells, and transendothelial migration of GIST cells. GIST-T1 cells with high $C A D M-1$ expression induced by $C A D M 1$ cDNA transfection, when compared to the original GIST-T1 cells with very low CADM1 expression, had decreased ability to grow, migrate, and invade but increased ability to adhere to endothelial cells and emigrate by transendothelial migration. CADM1 might play a role in tumor metastasis by facilitating adhesion between vascular endothelial cells and GIST cells and subsequent transendothelial migration of GIST cells with high $C A D M 1$ expression. There is a possibility that the higher expression of $C A D M 1$ in small intestinal GISTs than in gastric GISTs could contribute to the higher metastatic rate of small intestinal GISTs. CADM1 might provide a new strategy for inhibiting metastasis of small intestinal GISTs.

\section{Materials and methods}

Tumor tissue samples. Fresh tissue samples of a representative gastric GIST and a representative small intestinal GIST were collected intraoperatively, frozen, and stored at $-80^{\circ} \mathrm{C}$ until use. RNA and protein were extracted from the samples. The RNA and protein in the present experiments have been used in previous experiments (10).

Cell lines. A human GIST cell line, GIST-T1, which is derived from a metastatic pleural tumor from gastric GIST in a Japanese woman, was purchased from Cosmo Bio. It harbors a heterozygous c-kit gene mutation at exon 11 (an in-frame deletion of 19 amino acids from Val560 to Tyr578). The GIST-T1 cell line was maintained in Dulbecco's modified Eagle's medium (Sigma-Aldrich; Merck KGaA) supplemented with 10\% fetal bovine serum (FBS) (BioWest), $100 \mathrm{U} / \mathrm{ml}$ of penicillin $\mathrm{G}$ and $100 \mu \mathrm{g} / \mathrm{ml}$ of streptomycin (Invitrogen; Thermo Fisher Scientific) at $37^{\circ} \mathrm{C}$ in $5 \% \mathrm{CO}_{2}$. Human umbilical vein endothelial cells (HUVECs) (Takara) were grown in Endothelial Cell Growth Medium 2 (Takara).

CADM1 cDNA transfection into GIST-T1 cells. Full length of $C A D M 1$ cDNA was amplified by the reverse transcriptase polymerase chain reaction (PCR) using the primers listed in Table SI, Ampli Taq Gold (Thermo Fisher Scientific), and mRNA extracted from a small intestinal GIST highly expressing $C A D M 1$ mRNA. Amplified DNA fragment was electrophoresed and collected. After cutting with the EcoRI and XhoI enzymes, it was subcloned into EcoRI and XhoI sites of the pcDNA ${ }^{\mathrm{TM}}$ 3.1/Zeo (+) mammalian expression vector (Thermo Fisher Scientific). To verify product authenticity, the obtained vector with $C A D M 1$ cDNA was sequenced using ABI BigDye Terminator ver. 3.1 (Applied Biosystems; Thermo Fisher Scientific) and an ABI Prism 3100-Avant Genetic Analyzer (Applied Biosystems; Thermo Fisher Scientific). GIST-T1 cells $\left(1 \times 10^{6}\right)$ were suspended with $3 \mu \mathrm{g}$ of the vector with full-length $C A D M 1$ cDNA in $100 \mu \mathrm{l}$ of Cell Line Nucleofector kit V solution (Lonza) and electroporated using the Amaxa Nucleofector II machine (program T-030) (Lonza) according to the manufacturer's protocol. GIST-T1 cells stably expressing CADM1 (GIST-T1-CAD) were selected in $250 \mu \mathrm{g} / \mathrm{ml}$ of Zeocin (Thermo Fisher Scientific), and a monoclonal cell population was isolated by limiting dilution. All experiments using recombinant DNA were approved by the Committee for Recombinant DNA Experiments of Hyogo College of Medicine (no. 24015).

Western blotting. GIST-T1 cells, GIST-T1-CAD cells, a representative gastric GIST tissue, and a representative small intestinal GIST tissue were lysed in CelLytic M Cell Lysis Reagent (Sigma-Aldrich; Merck KGaA) containing $5 \mathrm{mM}$ NAF, $1 \mathrm{mM} \mathrm{Na}_{3} \mathrm{VO}_{4}$, and proteinase inhibitor cocktail (Roche). As described previously (10), almost all gastric GISTs express a very low level of CADM1 protein and almost all small intestinal GISTs apparently express CADM1 protein. Western blot analysis was performed as previously reported (10). Briefly, anti-CADM1 chicken monoclonal antibody (clone. 3E1, MBL International), anti-KIT rabbit polyclonal antibody (A4502, Dako) or anti- $\beta$-actin mouse monoclonal antibody (ab8226, Abcam) were used for primary antibodies after electrophoresis and membrane transfer. Then, membranes were incubated either with horse radish peroxidase (HRP)-conjugated donkey anti-chicken IgY antibody (EMD Millipore, Sigma-Aldrich; Merck KGaA), HRP-conjugated goat anti-rabbit IgG antibody, or HRP-conjugated goat anti-mouse IgG antibody (Dako) after the electrophoresis and membrane transfer. Proteins of interest were then visualized by incubation with enhanced chemiluminescence (ECL) reagent (Promega).

Real Time (RT)-quantitative ( $q$ ) PCR. Total RNA was extracted from GIST-T1 cells, GIST-T1-CAD cells, a representative gastric GIST tissue, and a representative small intestinal GIST tissue using RNeasy mini kit (Qiagen, Inc.), and $10 \mu \mathrm{g}$ of total RNA was applied for RT-qPCR templates. As described previously (10), almost all gastric GISTs express a very low level of CADMI mRNA and almost all small intestinal GISTs apparently express $C A D M 1$ mRNA. TaqMan RT-qPCR analysis was performed using the Applied Biosystems STEP $\mathrm{ONE}^{\mathrm{TM}}$ standard real-time PCR system (Applied Biosystems; Thermo Fisher Scientific) and sets of primers and probes of Human CADM1 (Hs00204937_m1) and Human GAPDH (Hs99999905_m1) in TaqMan ${ }^{\mathrm{TM}}$ Gene Expression Assay (4331182, Applied Biosystems; Thermo Fisher Scientific) according to the manufacturer's instructions.

Cell proliferation assay. GIST-T1 and GIST-T1-CAD cells were plated in 24-well plates (Corning Incorporated) at $2 \times 10^{4}$ cells per well in growth medium. After incubation for $1,3,5$, and 7 days, cells were trypsinized, resuspended in Accumax (Innovative Cell Technologies), and counted by hemocytometer (Z1, Beckman Coulter). Six wells were used for each cell type in each experiment. The cell proliferation assay was repeated three times.

Migration assay. Migration ability was assessed by the wound-healing assay. GIST-T1 and GIST-T1-CAD cells were 
seeded in 6-well plates at $3 \times 10^{5}$ cells and allowed to grow to $90 \%$ confluence. The cell monolayer was scratched with a sterile micropipette tip, and then serum-free medium was added into plates after washing the cells thrice with PBS. Photographs of images captured at $\times 200$ magnification were taken at the same six selected locations for each well under a phase contrast microscope (All-in-One Microscope; Keyence). The area that remained clear after $0,1,2,3$, and 4 days was quantified with ImageJ (National Institutes of Health) and the covered area was calculated by comparing to the area of the wound at day 0 . This assay was repeated three times.

Migration and invasion assays. The migration assay was performed using Falcon cell culture inserts (Corning Incorporated) without Matrigel and the invasion assay was performed using 24-well BD Bio-Coat Matrigel Invasion Chambers (BD Biosciences) according to the manufacturer's protocol. GIST-T1 and GIST-T1-CAD cells were resuspended at a density of $5 \times 10^{5}$ cells $/ 1$ in $0.5 \mathrm{ml}$ of the serum-free medium and added into the upper chamber of the insert. DMEM supplemented with $10 \%$ FBS $(0.75 \mathrm{ml})$ was added to the lower chambers. After incubation for 2 days, non-migrated or non-invaded cells were removed from the upper surface of the membranes using a cotton tipped swab. The cells adhering to the bottom surface of the membrane were fixed and permeabilized in $10 \%$ neutral formalin and $100 \%$ methanol, respectively. Migrated or invaded cells were stained by Giemsa staining and counted in nine selected microscope fields per membrane. The experiments were conducted three times.

Tumor-endothelial cell adhesion assay. Static adhesion assay using fluorescence-labeled tumor cells was performed. HUVECs $\left(2.5 \times 10^{5}\right.$ cells/well $)$ pretreated with or without $10 \mathrm{ng} / \mathrm{ml} \mathrm{TNF- \alpha} \mathrm{(Invitrogen;} \mathrm{Thermo} \mathrm{Fisher} \mathrm{Scientific)}$ were cultured in 96-well plates overnight. TNF- $\alpha$ has the potential to stimulate endothelial cell adhesion. GIST-T1 and GIST-T1-CAD cells were labeled with $2 \mu \mathrm{g} / \mathrm{ml}$ Calcein-AM (Dōjindo Laboratories) at $37^{\circ} \mathrm{C}$ for $30 \mathrm{~min}$, washed thrice with PBS, and resuspended at $2.5 \times 10^{6}$ cells $/ \mathrm{ml}$ with serum-free DMEM, and followed by pipetting onto confluent HUVECs monolayers. After coculturing for $2 \mathrm{~h}$, medium and unbounded tumor cells were removed and discarded. Adherent tumor cells and endothelial cells were washed three times with PBS. Then the amount of Calcein-AM fluorescence was measured using a fluorescence microplate reader (2030 ARVO X4, PerkinElmer Life and Analytical Sciences), at an excitation wavelength of $485 \mathrm{~nm}$ and emission wavelength of $530 \mathrm{~nm}$.

Transendothelial migration assay. HUVECs $\left(2 \times 10^{5}\right)$ pretreated with $10 \mathrm{ng} / \mathrm{ml} \mathrm{TNF-} \alpha$ were seeded onto 24 -well Transwell Inserts and cultured overnight. After formation of a confluent HUVEC monolayer, tumor cells labeled with Calcien-AM were added to the upper chamber, and cells were cocultured for $48 \mathrm{~h}$. After incubation, the non-migrated cells which were present on the upper side of the membrane were removed with a cotton tipped swab, and the transmigrated cells on the bottom side of the membrane were fixed with $10 \%$ neutral formalin. Transmigrated cells were visualized using a fluorescence microscope and counted from 10 random fields under x200 magnification. Experiments were performed in triplicate and repeated three times.

Statistical analysis. Statistical analysis of proliferation assay, wound-healing assay was performed by two-way mixed ANOVA followed by Bonferroni's multiple comparison test. The significance of cell migration and Matrigel invasion in transwell assay, adhesion assay and transendothelial migration assay was analyzed by unpaired Student t-test. $\mathrm{P}<0.01$ was considered statistically significant.

\section{Results}

GIST-T1-CAD cells show high expression of CADM1. GIST-T1 cells are a cell line of GIST cells originally derived from the stomach. Consistent with our previous report (10) showing that $C A D M 1$ expression was much weaker in GISTs of gastric origin than in GISTs of small intestinal origin, no CADM1 protein and $C A D M 1 \mathrm{mRNA}$ were detected in original GIST-T1 cells by western blotting and RT-qPCR, respectively (Fig. 1A and B). Using transfection of full length of $C A D M 1$ cDNA into GIST-T1 cells, we tried to establish GIST-T1 cells stably expressing CADM1 (GIST-T1-CAD cells). Western blotting and RT-qPCR, respectively, revealed high expression of CADM1 protein and CADM1 mRNA in the obtained GIST-T1-CAD cells (Fig. 1A and B).

High CADM1 expression suppresses the proliferation, migration, and invasion of cultured GIST cells. To investigate whether CADM1 is involved in the proliferation of GIST cells, we compared proliferative ability between GIST-T1 cells and GIST-T1-CAD cells. Cell number was counted at days $0,1,3,5$, and 7 after seeding with $2 \times 10^{4}$ of both cells. After day 3, the number of GIST-T1-CAD cells was significantly smaller than the number of GIST-T1 cells (Fig. 2) $(\mathrm{P}<0.001)$. To evaluate the effect of CADM1 on the ability of GIST cells to migrate, we performed a wound healing assay. Closure of scratches in GIST-T1 and GIST-T1-CAD cell monolayers was measured at 0,1 , 2, 3, and 4 days. Closure of scratches in GIST-T1-CAD cell monolayers was significantly slower than closure of scratches in GIST-T1 cell monolayers at 1,2,3, and 4 days (Fig. 3A and B) $(\mathrm{P}<0.001)$. We also examined the effect of CADM1 on migration of GIST cells through transwell membranes without Matrigel and invasion of GIST cells through transwell membranes coated with Matrigel. Fewer GIST-T1-CAD cells than GIST-T1 cells appeared to migrate through the transwell membrane (Fig. 4A and B), but the difference was not statistically significant $(\mathrm{P}=0.1416)$. On the other hand, statistically significantly fewer GIST-T1-CAD cells than GIST-T1 cells invaded the Matrigel-coated transwell membrane (Fig. 4C and D) $(\mathrm{P}<0.001)$.

High CADM1 expression augments adherence to human endothelial cells and transmigration of cultured GIST cells through human endothelium. To explore the effect of CADM1 on GIST cell-vascular endothelial cell adhesion, we performed a static adhesion assay based on the binding of fluorescence-labeled GIST-T1 cells and fluorescence-labeled GIST-T1-CAD cells to HUVECs 

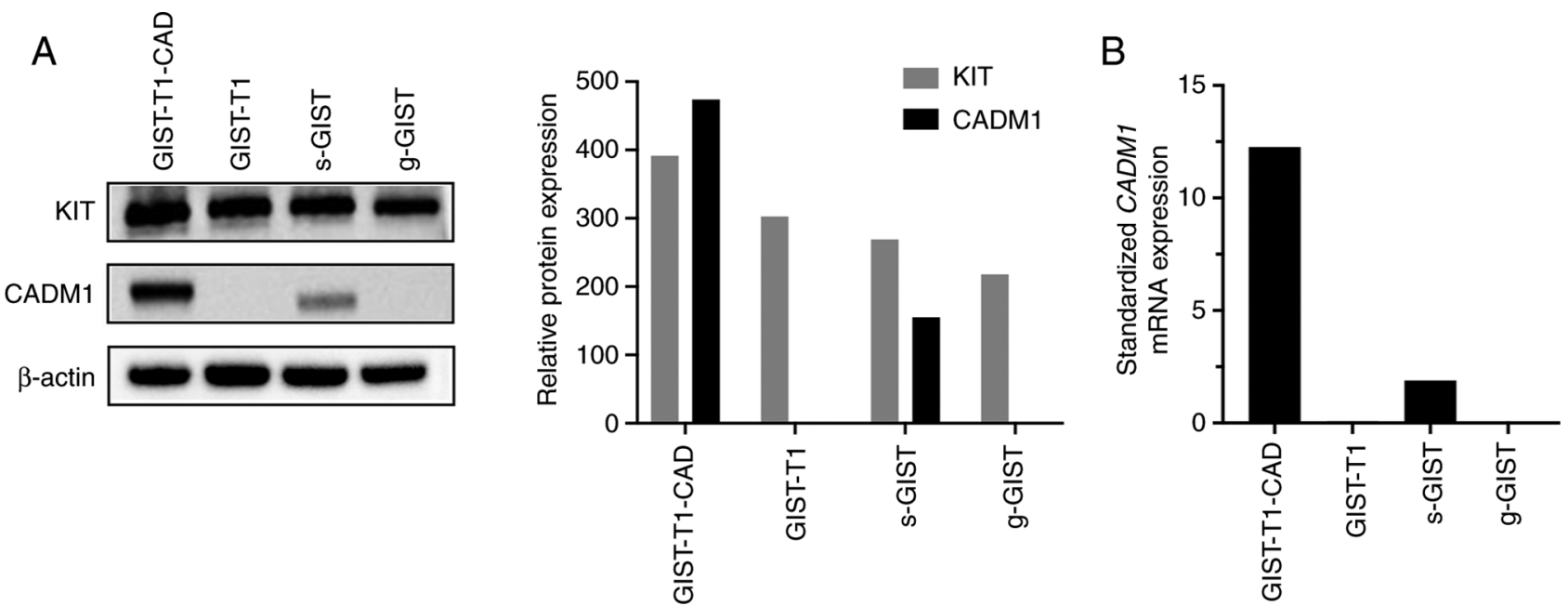

Figure 1. GIST-T1-CAD cells show high expression of CADM1. (A) The relative protein level of CADM1 in GIST-T1-CAD and GIST-T1 cells, and s-GIST and g-GIST tissues was examined by western blotting. (B) Level of CADM1 mRNA expression was examined via reverse transcription-quantitative PCR and standardized by GAPDH mRNA expression. Original GIST-T1 cells and g-GIST tissue did not show apparent CADM1 expression, but GIST-T1-CAD cells stably transfected with $C A D M 1$ cDNA and the s-GIST tissue showed distinct CADM1 expression. GIST, gastrointestinal stromal tumor; GIST-T1-CAD, GIST-T1 cells with high $C A D M 1$ expression induced by $C A D M 1$ cDNA transfection; CADM1, cell adhesion molecule 1; s-GIST, small intestinal GIST; g-GIST, gastric GIST; KIT, receptor tyrosine kinase encoded by c-kit.

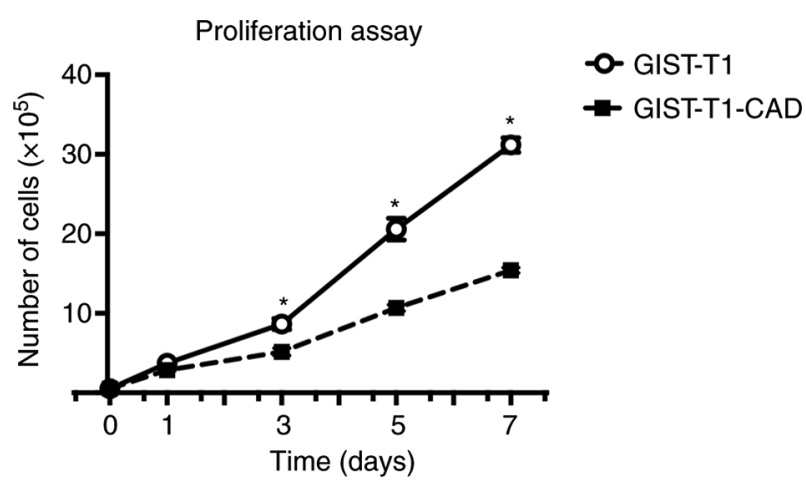

Figure 2. CADM1 expression inhibits GIST-T1 cell growth in vitro. The cells were seeded at $2 \times 10^{4}$ cells $/ \mathrm{ml}$ in 24 -well plates and the number of cells was counted at days $1,3,5$ and 7 . The number of GIST-T1-CAD cells was less than that of the original GIST-T1 cells. Statistical significance $\left({ }^{*} \mathrm{P}<0.001\right.$ vs. GIST-T1 CAD cells at each time point, $\mathrm{n}=6$, two-way mixed ANOVA followed by Bonferroni's post hoc test) was observed at days 3,5 and 7. GIST, gastrointestinal stromal tumor; GIST-T1-CAD, GIST-T1 cells with high $C A D M 1$ expression induced by $C A D M 1$ cDNA transfection; CADM1, cell adhesion molecule 1.

monolayers. We firstly examined the ability of tumor cells to adhere to TNF- $\alpha$-unstimulated endothelium. GIST-T1-CAD cell-HUVEC adherence was 16.6 times greater than GIST-T1 cell-HUVEC adherence (Fig. 5A) $(\mathrm{P}<0.001)$. After overnight stimulation of HUVECs by TNF- $\alpha(10 \mathrm{ng} / \mathrm{ml}$ for $12 \mathrm{~h})$, the number of GIST-T1-CAD cells adhering to HUVECs and GIST-T1 cells adhering to HUVECs was augmented. Even after stimulation of HUVECs by TNF- $\alpha$, the number of GIST-T1-CAD cells adhering to HUVECs was 3 times greater than that of GIST-T1 cells adhering to HUVECs (Fig. 5B) $(\mathrm{P}<0.0001)$. To determine the effect of CADM1 on GIST cell transmigration across endothelium, the transendothelial migration assay was performed using TNF- $\alpha$-stimulated HUVECs, GIST-T1 cells, and GIST-T1-CAD cells. Number of migrated Calcein-AM-labeled GIST-T1-CAD cells was significantly

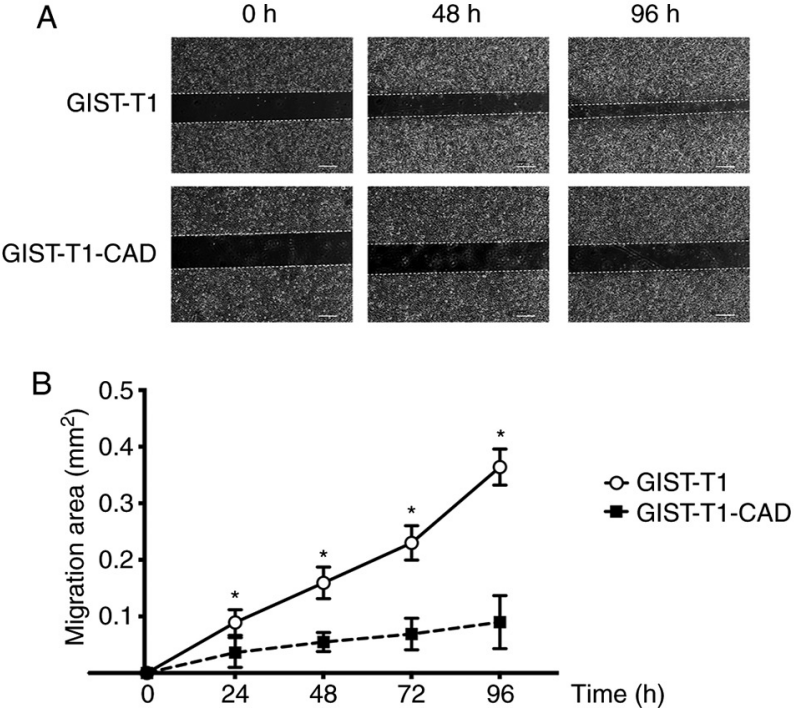

Figure 3. CADM1 expression impairs cell motility and migration of GIST-T1 cells in vitro. (A) Wound-healing assay showed that GIST-T1-CAD cell migration was decreased compared with the migration of GIST-T1 cells. The images show representative results at days 0,2 and 4. Scale bar, $200 \mu \mathrm{m}$. (B) The calculated area of migration is shown. Statistical significance $\left({ }^{*} \mathrm{P}<0.001\right.$ vs. GIST-T1 CAD cells at each time point, $n=9$, two-way mixed ANOVA followed by Bonferroni's post hoc test) was observed at days 1, 2, 3 and 4. GIST, gastrointestinal stromal tumor; GIST-T1-CAD, GIST-T1 cells with high $C A D M 1$ expression induced by $C A D M 1$ cDNA transfection; CADM1, cell adhesion molecule 1 .

larger than the number of migrated Calcein-AM-labeled GIST-T1 cells (Fig. 6A and B) $(\mathrm{P}<0.001)$.

\section{Discussion}

We have recently reported that $C A D M 1$ is expressed specifically in most small intestinal GISTs but not in most gastric GISTs (10). Patients with small intestinal GISTs are 
A

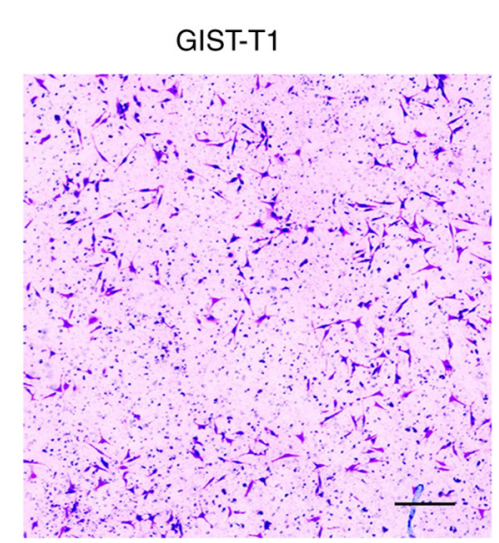

C

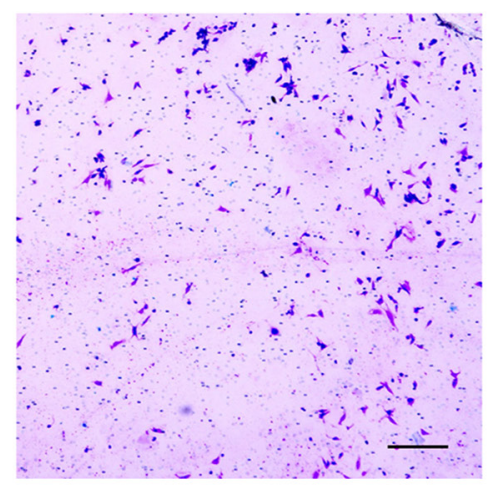

GIST-T1-CAD
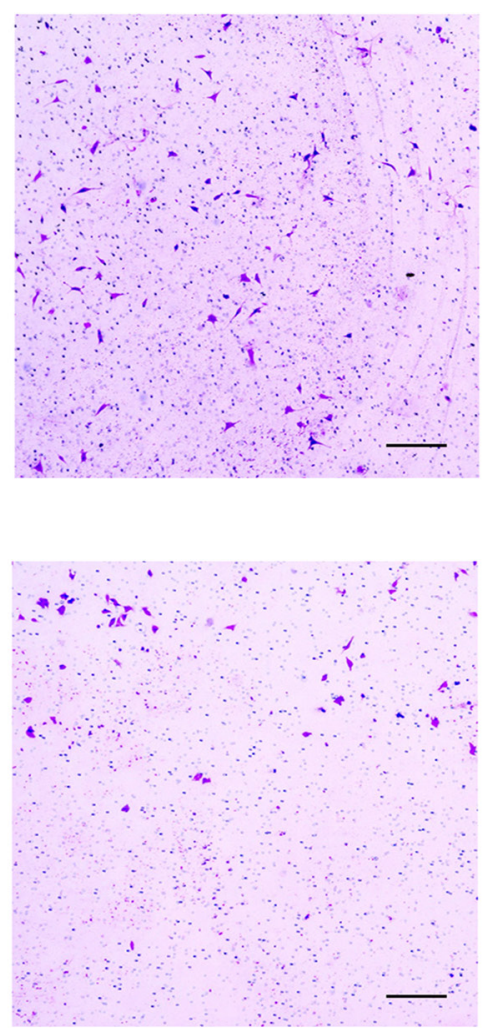

$\mathrm{B}$
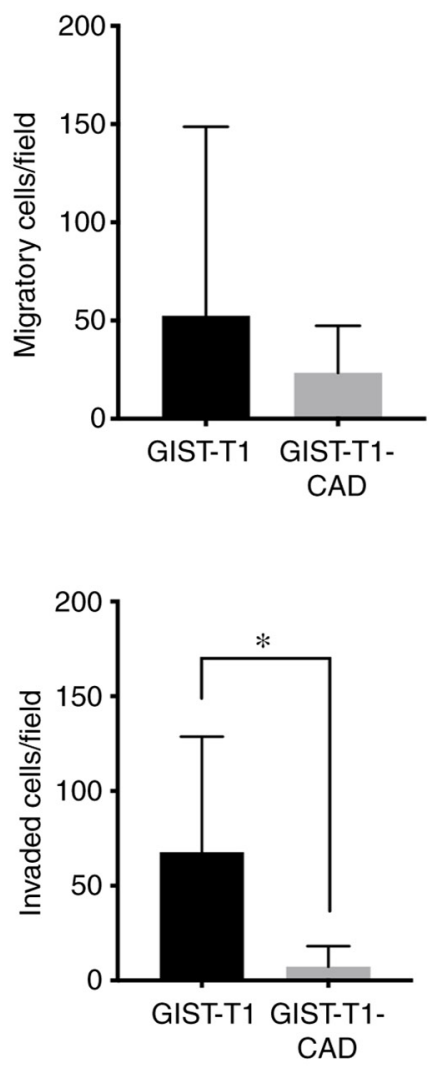

Figure 4. CADM1 expression suppresses GIST-T1 cell migration and invasion in vitro. Representative images of cell (A) migration and (C) Matrigel invasion in Transwell assays using the original GIST-T1 cells and GIST-T1-CAD cells. Scale bar, $200 \mu \mathrm{m}$. There were more migrating and invading original GIST-T1 cells than migrating and invading GIST-T1-CAD cells. The means of nine random microscopic fields per membrane in the cell (B) migration and (D) Matrigel invasion assays were calculated. Statistical significance ( ${ }^{*} \mathrm{P}<0.001, \mathrm{n}=3$, unpaired Student's t-test) is shown. GIST, gastrointestinal stromal tumor; GIST-T1-CAD, GIST-T1 cells with high $C A D M 1$ expression induced by $C A D M 1$ cDNA transfection; CADM1, cell adhesion molecule 1.

A

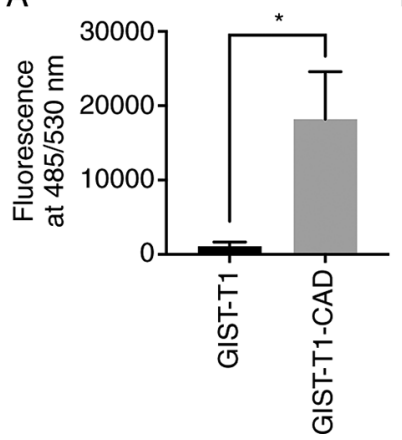

B

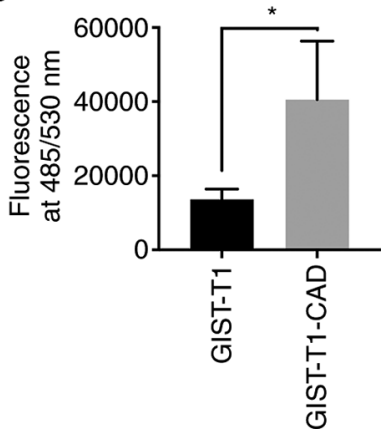

A

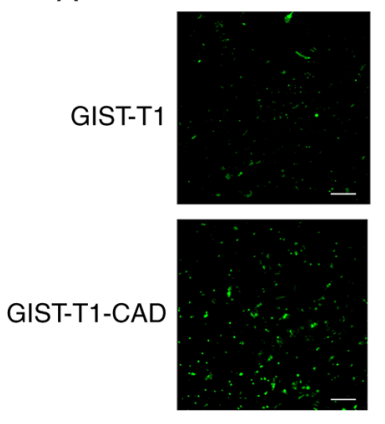

B

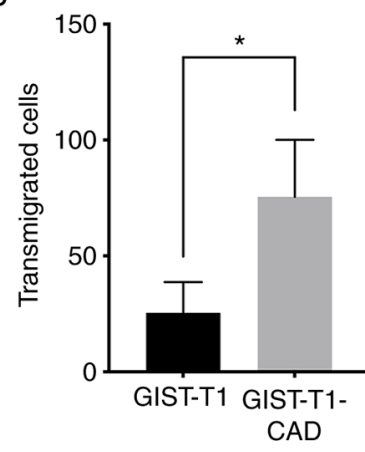

Figure 5. CADM1 expression enhances adhesion of GIST-T1 cells to endothelial cells. (A) Without TNF- $\alpha$ stimulation, significantly greater numbers of Calcein-AM labeled GIST-T1-CAD cells than Calcein-AM labeled original GIST-T1 cells adhered to HUVECs. (B) With TNF- $\alpha$ stimulation, significantly greater numbers of Calcein-AM labeled GIST-T1-CAD cells than Calcein-AM labeled original GIST-T1 cells also adhered to HUVECs. Statistical significance ( $(\mathrm{P}<0.001, \mathrm{n}=21$, unpaired Student's t-test) is shown GIST, gastrointestinal stromal tumor; GIST-T1-CAD, GIST-T1 cells with high $C A D M 1$ expression induced by $C A D M 1$ cDNA transfection; CADM1, cell adhesion molecule 1; HUVECs, human umbilical vein endothelial cells.

considered to have a worse prognosis than patients with gastric GISTs because of their higher risk of metastases and tumor-related death (7). Therefore, we tried to clarify whether high CADM1 expression in small intestinal GISTs affects
Figure 6. CADM1 expression enhances transendothelial migration of GIST-T1 cells. (A) Representative images of transmigrated GIST-T1 cells and GIST-T1-CAD cells labeled by Calcein-AM green dye are shown. Scale bar, $200 \mu \mathrm{m}$. (B) The mean numbers of GIST-T1-CAD cells and GIST-T1 cells in 14 random microscopic fields per membrane are shown. The number of GIST-T1-CAD cells showing transendothelial migration was significantly higher than that of GIST-T1 cells showing transendothelial migration. Data are from three independent experiments. Statistical significance $\left({ }^{*} \mathrm{P}<0.001\right.$, $\mathrm{n}=14$, unpaired Student's t-test) is shown. GIST, gastrointestinal stromal tumor; GIST-T1-CAD, GIST-T1 cells with high $C A D M 1$ expression induced by $C A D M 1$ cDNA transfection; CADM1, cell adhesion molecule 1 .

the biological behavior of GISTs. In the present study, proliferation, migration, invasion, adhesion to endothelial cells, and transendothelial migration were compared between 
original GIST-T1 cells with very low $C A D M 1$ expression and GIST-T1 cells with high CADMI expression induced by $C A D M 1$ cDNA transfection (GIST-T1-CAD cells). GIST-T1-CAD cells showed lower ability to grow, migrate, and invade, but higher ability to adhere to endothelium and transmigrate across endothelium than the original GIST-T1 cells. These results suggested that CADM1 might facilitate the metastasis of GISTs by increasing tumor cell adherence to vascular endothelial cells and subsequent passage through the vascular endothelium but not by increasing tumor cell growth and motility.

$C A D M 1$ expression is frequently lost in numerous types of epithelial neoplasms (13-15), and CADM1 is considered to be a tumor suppressor in epithelial neoplasms. In contrast, upregulated $C A D M 1$ appears to promote ATLL (16) and AML (17) progression through enhancement of tumor cell growth, tissue invasion, and adhesion to the vascular endothelium (18). There is also recent research that high CADMI expression in SCLCs might promote the malignant features of the cancer $(19,20)$. In the present study, we showed that high $C A D M 1$ expression decreased tumor cell growth and motility but increased tumor cell adherence to vascular endothelial cells and subsequent transmigration across vascular endothelium. Thus, high CADM1 expression in GISTs appears to have two roles, as a tumor suppressor and a tumor promoter. Poorer prognosis in small intestinal GISTs might indicate that the metastasis promoter role through strong adherence to endothelial cells exceeds the tumor suppressor role through reduced tumor growth and motility.

Expression of $C A D M 1$ is extremely low in most gastric GISTs (10). The loss of $C A D M 1$ expression frequently found in a variety of cancers is considered to be due to aberrant hypermethylation of the $C A D M 1$ promoter. In our preliminary research, there was no methylation of $C A D M 1$ promoter in not only small intestinal GISTs but also gastric GISTs (unpublished data), and methylation is not considered to be the cause of low $C A D M 1$ expression in gastric GISTs. Therefore, we should clarify the cause of low CADM1 expression in gastric GISTs.

In the present study, expression of $C A D M 1$ in cultured GIST cells increased their ability to adhere to endothelium and transmigrate across endothelium. This is similar to previous reports showing CADM1 promotes ATLL cell infiltration of organs (18). CADM1 promotes an invasive phenotype of ATLL cells by activating the Rac pathway through PDZ-BM interaction with TIAM1 (21). A recent study reported that CADM1 recruits 4.1R to the cell-cell contact site and can enhance the malignant features of SCLC (20). In addition, 4.1R modulates the localization of several G-protein coupled receptors including Duffy/ACKR1 (22). Besides, it had been reported that CADM1 activates PI3K signaling by forming a tripartite protein complex with the p85 subunit of PI3K through the membrane-associated guanylate kinases (MAGuKs), membrane palmitoylated protein 3 (MPP3) and Drosophila tumor suppressor discs large (Dlg) (23), which play roles in the extension of epithelial cells. Further examination of CADM1 involvement in the mechanism of GIST cell adhesion to endothelium and transmigration of GIST cells across endothelium is required.
$C A D M 1$ is ubiquitously expressed in vascular endothelial cells (24). In our study, compared to the original GIST-T1 cells, GIST-T1-CAD cells showed a much higher ability to adhere to TNF- $\alpha$ unstimulated HUVECs, suggesting that CADM1-mediated homotypic contacts between GIST-T1-CAD cells and HUVECs is extremely important for their adhesion. The adhesion of the original GIST-T1 cells to HUVECs was significantly enhanced by pre-stimulation of the HUVECs with TNF- $\alpha$. TNF- $\alpha$ can upregulate expression of the intercellular adhesion molecule type 1 (ICAM-1), E-selectin, and vascular cell adhesion molecule type 1 $(V C A M-1)(25,26)$ in vascular endothelial cells. Promotion of original GIST-T1 cell adhesion to TNF- $\alpha$-stimulated HUVECs might be significantly induced by increased expression of those adhesion molecules on TNF- $\alpha$ stimulated HUVECs. On the other hand, improved GIST-T1-CAD cell adhesion to HUVECs after TNF- $\alpha$-stimulation might derive from increased expression of not only those adhesion molecules but also CADM1 on TNF- $\alpha$-stimulated HUVECs. Detailed mechanisms underlying the change in the ability of GIST-T1-CAD cells and original GIST-T1 cells to adhere to HUVECs before and after TNF- $\alpha$ stimulation should be clarified.

Recently, anti-CADM1 antibodies were developed as a promising candidate agent for reducing ATLL cell invasion via blocking cell adhesion. The antibodies appear to show a minimal cytotoxic effect on the growth of the ATLL cell line (27). Such antibodies are also expected to inhibit GIST cell adhesion to the endothelium and show inhibition of GIST metastasis. Moreover, anti-CADM1 antibodies could cause cell damage via antibody dependent-cellular cytotoxicity or complement-dependent cytotoxicity. The anti-tumor effect of antibody-drug conjugates using CADM1 antibodies might also be stronger. Anti-CADM1 antibodies could become a new treatment strategy for small intestinal GISTs.

There are some limitations in our study. First, we did not examine expression of surface adhesion molecules other than $C A D M 1$ that may affect both migration/invasion of GIST-T1 cells and adhesion between GIST-T1 cells and HUVECs. CADM1 cDNA transfection to GIST-T1 cells may also change the expression of such surface adhesion molecules. We are planning to examine the expression levels of those molecules in HUVECs and GIST-T1 cells before and after $C A D M 1$ cDNA transfection. Second, we did not examine that $C A D M 1$ expression in HUVECs was really augmented after TNF- $\alpha$ stimulation. We will examine whether expression levels of not only CADM1 but also other surface adhesion molecules increase in HUVECs after TNF- $\alpha$ stimulation in the near future. Third, we only carried out in vitro experiments concerning migration, invasion and adhesion of GIST-T1 and GIST-T1-CAD cells, but CADM1 contribution to metastatic activity in GIST should be evaluated by in vivo experiments. In vivo studies using mouse models of metastasis and GIST cells are being planned.

In summary, CADM1 in GISTs might act as a suppressor of tumor growth, migration, and matrix invasion, but stronger GIST cell-endothelial cell interaction induced by high $C A D M 1$ expression could serve as a potential target for the treatment of small intestinal GISTs, especially for inhibiting GIST metastasis. 


\section{Acknowledgements}

Not applicable.

\section{Funding}

No funding was received.

\section{Availability of data and materials}

All data generated or analyzed during this study are included in this published article.

\section{Authors' contributions}

JY, TK, NK, TY, MY, KI, AI and SH participated in data collection and discussion of the findings. JY, TK, NK, TY and MY carried out the experiments. JY and TK performed the statistical analysis. JY and SH wrote the manuscript. JY and $\mathrm{SH}$ confirm the authenticity of all the raw data. All authors have read and approved the final manuscript.

\section{Ethics approval and consent to participate}

Experiments using recombinant DNA were approved by the Committee for Recombinant DNA Experiments of Hyogo College of Medicine (approval no. 24015; Nishinomiya, Japan). The use of fresh human gastrointestinal stromal tumor (GIST) tissue samples for GIST assays, including gene analysis, was approved by the Ethical Committee of Hyogo College of Medicine (approval no. 28), and the patients/participants provided their written informed consent to participate in this study.

\section{Patient consent for publication}

Not applicable.

\section{Competing interests}

The authors declare that they have no competing interests.

\section{References}

1. Hirota S, Isozaki K, Moriyama Y, Hashimoto K, Nishida T, Ishiguro S, Kawano K, Hanada M, Kurata A, Takeda M, et al: Gain-of-function mutations of c-kit in human gastrointestinal stromal tumors. Science 279: 577-580, 1998.

2. Miettinen M and Lasota J: Gastrointestinal stromal tumors-definition, clinical, histological, immunohistochemical, and molecular genetic features and differential diagnosis. Virchows Arch 438: 1-12, 2001.

3. Heinrich MC, Corless CL, Duensing A, McGreevey L, Chen CJ, Joseph N, Singer S, Griffith DJ, Haley A, Town A, et al: PDGFRA activating mutations in gastrointestinal stromal tumors. Science 299: 708-710, 2003.

4. Kinoshita K, Hirota S, Isozaki K, Ohashi A, Nishida T, Kitamura Y, Shinomura Y and Matsuzawa Y: Absence of c-kit gene mutations in gastrointestinal stromal tumours from neurofibromatosis type 1 patients. J Pathol 202: 80-85, 2004.

5. Agaram NP, Wong GC, Guo T, Maki RG, Singer S, Dematteo RP, Besmer P and Antonescu CR: Novel V600E BRAF mutations in imatinib-naive and imatinib-resistant gastrointestinal stromal tumors. Genes Chromosomes Cancer 47: $853-859,2008$
6. Pantaleo MA, Astolfi A, Urbini M, Nannini M, Paterini P, Indio V, Saponara M, Formica S, Ceccarelli C, Casadio R, et al: Analysis of all subunits, SDHA, SDHB, SDHC, SDHD, of the succinate dehydrogenase complex in KIT/PDGFRA wild-type GIST. Eur J Hum Genet 22: 32-39, 2014.

7. Emory TS, Sobin LH, Lukes L, Lee DH and O'Leary TJ: Prognosis of gastrointestinal smooth-muscle (stromal) tumors: Dependence on anatomic site. Am J Surg Pathol 23: 82-87, 1999.

8. Yang Z, Wang F, Liu S and Guan W: Comparative clinical features and short-term outcomes of gastric and small intestinal gastrointestinal stromal tumours: A retrospective study. Sci Rep 9: 10033, 2019.

9. Antonescu CR, Viale A, Sarran L, Tschernyavsky SJ, Gonen M, Segal NH, Maki RG, Socci ND, DeMatteo RP and Besmer P: Gene expression in gastrointestinal stromal tumors is distinguished by KIT genotype and anatomic site. Clin Cancer Res 10: 3282-3290, 2004.

10. Yuan J, Kihara T, Kimura N, Hashikura Y, Ohkouchi M, Isozaki K, Takahashi T, Nishida T, Ito A and Hirota S: Differential expression of CADM1 in gastrointestinal stromal tumors of different sites and with different gene abnormalities. Pathol Oncol Res 27: 602008, 2021.

11. Biederer T, Sara Y, Mozhayeva M, Atasoy D, Liu X, Kavalali ET and Sudhof TC: SynCAM, a synaptic adhesion molecule that drives synapse assembly. Science 297: 1525-1531, 2002.

12. Kuramochi M, Fukuhara H, Nobukuni T, Kanbe T, Maruyama T, Ghosh HP, Pletcher M, Isomura M, Onizuka M, Kitamura T, et al: TSLC1 is a tumor-suppressor gene in human non-small-cell lung cancer. Nat Genet 27: 427-430, 2001

13. Allinen M, Peri L, Kujala S, Lahti-Domenici J, Outila K, Karppinen SM, Launonen V and Winqvist R: Analysis of 11q21-24 loss of heterozygosity candidate target genes in breast cancer: Indications of TSLC1 promoter hypermethylation. Genes Chromosomes Cancer 34: 384-389, 2002.

14. Honda T, Tamura G, Waki T, Jin Z, Sato K, Motoyama T, Kawata S, Kimura W, Nishizuka S and Murakami Y: Hypermethylation of the TSLC1 gene promoter in primary gastric cancers and gastric cancer cell lines. Jpn J Cancer Res 93: 857-860, 2002 .

15. Zeng D, Wu X, Zheng J, Zhuang Y, Chen J, Hong C, Zhang F, Wu M and Lin D: Loss of CADM1/TSLC1 expression is associated with poor clinical outcome in patients with esophageal squamous cell carcinoma. Gastroenterol Res Pract 2016: 6947623, 2016.

16. Sasaki H, Nishikata I, Shiraga T, Akamatsu E, Fukami T, Hidaka T, Kubuki Y, Okayama A, Hamada K, Okabe H, et al: Overexpression of a cell adhesion molecule, TSLC1, as a possible molecular marker for acute-type adult T-cell leukemia. Blood 105: 1204-1213, 2005.

17. Fisser MC, Rommer A, Steinleitner K, Heller G, Herbst F, Wiese M, Glimm H, Sill H and Wieser R: Induction of the proapoptotic tumor suppressor gene cell adhesion molecule 1 by chemotherapeutic agents is repressed in therapy resistant acute myeloid leukemia. Mol Carcinog 54: 1815-1819, 2015.

18. Dewan MZ, Takamatsu N, Hidaka T, Hatakeyama K, Nakahata S, Fujisawa J, Katano H, Yamamoto N and Morishita K: Critical role for TSLC1 expression in the growth and organ infiltration of adult T-cell leukemia cells in vivo. J Virol 82: 11958-11963, 2008.

19. Kikuchi S, Iwai M, Sakurai-Yageta M, Tsuboi Y, Ito T, Maruyama T, Tsuda H, Kanai Y, Onizuka M, Sato Y and Murakami Y: Expression of a splicing variant of the CADM1 specific to small cell lung cancer. Cancer Sci 103: 1051-1057, 2012.

20. Funaki T, Ito T, Tanei ZI, Goto A, Niki T, Matsubara D and Murakami Y: CADM1 promotes malignant features of small-cell lung cancer by recruiting 4.1R to the plasma membrane. Biochem Biophys Res Commun 534: 172-178, 2020.

21. Masuda M, Maruyama T, Ohta T, Ito A, Hayashi T, Tsukasaki K, Kamihira S, Yamaoka S, Hoshino H, Yoshida T, et al: CADM1 interacts with Tiam1 and promotes invasive phenotype of human T-cell leukemia virus type I-transformed cells and adult T-cell leukemia cells. J Biol Chem 285: 15511-15522, 2010.

22. Baines AJ, Lu HC and Bennett PM: The Protein 4.1 family: Hub proteins in animals for organizing membrane proteins. Biochim Biophys Acta 1838: 605-619, 2014.

23. Murakami S, Sakurai-Yageta M, Maruyama T and Murakami Y: Trans-homophilic interaction of CADM1 activates PI3K by forming a complex with MAGuK-family proteins MPP3 and Dlg. PLoS One 9: e82894, 2014 
24. Tatsumi K, Taatjes DJ, Wadsworth MP, Bouchard BA and Bovill EG: Cell adhesion molecule 1 (CADM1) is ubiquitously present in the endothelium and smooth muscle cells of the human macro- and micro-vasculature. Histochem Cell Biol 138: $815-820,2012$

25. Ross EA, Douglas MR, Wong SH, Ross EJ, Curnow SJ, Nash GB, Rainger E, Scheel-Toellner D, Lord JM, Salmon M and Buckley CD: Interaction between integrin alpha9beta1 and vascular cell adhesion molecule-1 (VCAM-1) inhibits neutrophil apoptosis. Blood 107: 1178-1183, 2006.

26. Sana TR, Janatpour MJ, Sathe M, McEvoy LM and McClanahan TK: Microarray analysis of primary endothelial cells challenged with different inflammatory and immune cytokines. Cytokine 29: 256-269, 2005.
27. Chilmi S, Nakahata S, Fauzi YR, Ichikawa T, Tani C, Suwanruengsri M, Yamaguchi R, Matsuura T, Kurosawa G and Morishita K: Development of anti-human CADM monoclonal antibodies as a potential therapy for adult T-cell leukemia/lymphoma. Int J Hematol 112: 496-503, 2020.

This work is licensed under a Creative Commons Attribution-NonCommercial-NoDerivatives 4.0 International (CC BY-NC-ND 4.0) License. 